\title{
Interreflection coefficients for interior lighting calculations based on CIE methods
}

\author{
Masayuki IIZUKA* and kōzō ISHINO*
}

\begin{abstract}
The concept and practicability of two useful methods reported for interior lighting calculations in France have been widely introduced and discussed by a few Japanese investigators from various points of view CIE Publication No. 40 : "Calculations for Interior Lighting - Basic Method-"' was published in 1978, and the final report on "Applied Method" has now been prepared under the chairman of TC-1.5, A.B. de Graaff.

The authors compare the computation process and its characteristics of RM and MR coefficients obtained by the present studies with that of these coefficients based on the CIE methods approved by TC-1.5, and point out the difference between the data of RM and MR coefficients prepared in the CIE methods and the numerical data calculated by the authors. Lastly, they discuss the effect on the final computation results for lighting designs from the standpoints such as room index, suspension ratio and effective reflectance of the ceiling cavity.
\end{abstract}

\section{Introduction}

The concept and practicability of two useful methods reported already for interior lighting calculations in France have been widely introduced and discussed by a few Japanese investigators from various points of view ${ }^{1)}$. The two recent recommended methods for interior lighting calculations approved by CIE Technical Committee 1.5 intend to retain and combine all the good features of various methods. CIE Publication No. 40: "Calculations for Interior Lighting-Basic Method-" was published in $1978^{2)}$, and the final report on "Applied Method" has now been prepared under the chairman of TC-1.5, A.B. de Graaff ${ }^{3)}$.

The authors, first of all, review the characteristics and the roles in the CIE methods for computation of mean illuminance or reduced utilance and flux density of the installed luminaires by the linear expression. They lead up to the numerical computation formulas for obtaining the RM and MR coefficients by using the basic expression of luminous transfer which describes the general interreflection systems in an idealized room.

It is the purpose of this paper to compare the computation process and its characteristics of the RM and MR coefficients obtained by the present studies with those of these coefficients based on the CIE methods approved by TC-1.5, and to point out the difference between the data of the RM and MR

* Department of Electrical Engineering, Nagoya Institute of Technology, Gokiso-chō, Showa-ku, NAGOYA, 466 JAPAN coefficients prepared in the CIE methods and the numerical data calculated by the authors. Lastly, they discuss the effect on the final computation results for lighting designs from the standpoints such as room index, suspension ratio and effective reflectance of the ceiling cavity.

\section{Concept of CIE methods}

The CIE methods which contain both the "Basic Method" and the "Applied Method" can be used under the following conditions.

(a) Room is a rectangular parallelepiped,

(b) Working plane can be assumed to be practitical surface in place of floor,

(c) Room surfaces reflect uniformly and according to Lambert's law,

(d) Direct flux on working plane does not vary owing to direction of installed luminaires.

Main computation procedures of the CIE methods are as follows.

\section{(1) Cumulative zonal flux}

FC1, FC2, FC3 and FC4 correspond to cumulative zonal fluxes for the four zones of total solid angle: $\pi / 2, \pi, 3 \pi / 2,2 \pi$, respectively, and $F$ is the total flux of a luminaire.

\section{(2) Direct flux on the working plane}

The direct flux on the working plane emitted by a given luminaire or NM luminaires is calculated from the following expression by using "Geometric 
Multiplier" such as GM1, GM2, GM3 and GM4 prepared in the CIE methods. These GM coefficients depend on the position, mounting height and distribution curve of a luminaire.

$$
\begin{aligned}
F_{u j}= & F C 1 \cdot \sum_{i=1}^{4} G M 1_{i}+F C 2 \cdot \sum_{i=1}^{4} G M 2_{i} \\
& +F C 3 \cdot \sum_{i=1}^{4} G M 3_{i}+F C 4 \cdot \sum_{i=1}^{4} G M 4_{i} \cdots(1) \\
F 4= & \sum_{j=1}^{N M} F_{u j}
\end{aligned}
$$

where

$$
\left\{\begin{aligned}
F_{u j}: & \text { direct flux on working plane emitted } \\
& \text { by a luminaire } \\
F 4: & \text { direct flux on working plane emitted } \\
& \text { by total luminaires }
\end{aligned}\right.
$$

\section{(3) Flux density of the installed lumi- naires}

Installed luminaire flux per square metre floor area is defined as follows.

(a) Total flux density of the installed luminaires (NM) F/AB

(b) Downward fiux density of the installed luminaires (NM) FC4/AB

(c) Flux density emitted directly by the installations to the working plane $\mathrm{FA} / \mathrm{AB}$ where

$N, M$ : number of crosswise and lengthwise luminaires

$N M$ : number of total luminaires

$A, B$ : width and length of the room (working plane)

$A B$ : area of working plane

\section{(4) Calculations of mean illuminance}

The mean illuminance $E_{1}$ on the ceiling, $E_{3}$ on the walls and $E_{4}$ on the working plane are calculated by means of the linear functions of the flux density of the installed luminaires.

$$
\begin{aligned}
& E_{i}=R M_{i 1} \cdot(N M) F / A B+R M_{i 2} \cdot(N M) F C 4 / A B
\end{aligned}
$$

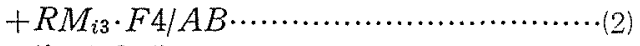

$$
\begin{aligned}
& (i=1,3,4)
\end{aligned}
$$

where, $R M_{i j}$ : $R M$ coefficients

$R M$ coefficients are called "Weighting Factors" or. "Interreflection Factors", and these values are given in the Tables in the CIE methods.

\section{(5) Calculations of flux density of installed luminaires}

By carrying out the inverse transformation of Eq. (2) $(N M) F / A B, \quad(N M) F C 4 / A B$ and $F 4 / A B$ are calculated by means of the following linear expression. For example:

$$
\begin{aligned}
(N M) F / A B= & M R_{11} \cdot E_{1}+M R_{13} \cdot E_{3} \\
& +M R_{14} \cdot E_{4} \quad \ldots \ldots \ldots \ldots
\end{aligned}
$$

where, $M R_{i j}: M R$ coefficients

$M R$ coefficients are given as well as $R M$ coeffi- cients in the Tables in the CIE methods. The main characteristics of the CIE methods are to carry out interior lighting calculations by using the $R M$ and $M R$ coefficients. It should be noted that mean illuminance on the frieze: $E_{2}$ defined as those parts of vertical surfaces which lie between the ceiling and the plane of the luminaires is approximately equal to mean illuminance on the ceiling: $E_{1}$.

\section{Induction procedure of RM and MR coefficients}

\subsection{RM coefficients for suspension ratio: $J=0$}

Fig. 1 shows the idealized room of a rectangular parallelepiped. The room surfaces contribute to interreflections of the radiation flux of installed luminaires. Total mean illuminance received by the surface: $i$ is equal to the sum of the direct mean illuminance and indirect mean illuminance obtained by interreflections. The basic expression of luminous transfer which describes the general interrefiection systems in an idealized room as shown in Fig. 1 is defined as follows.

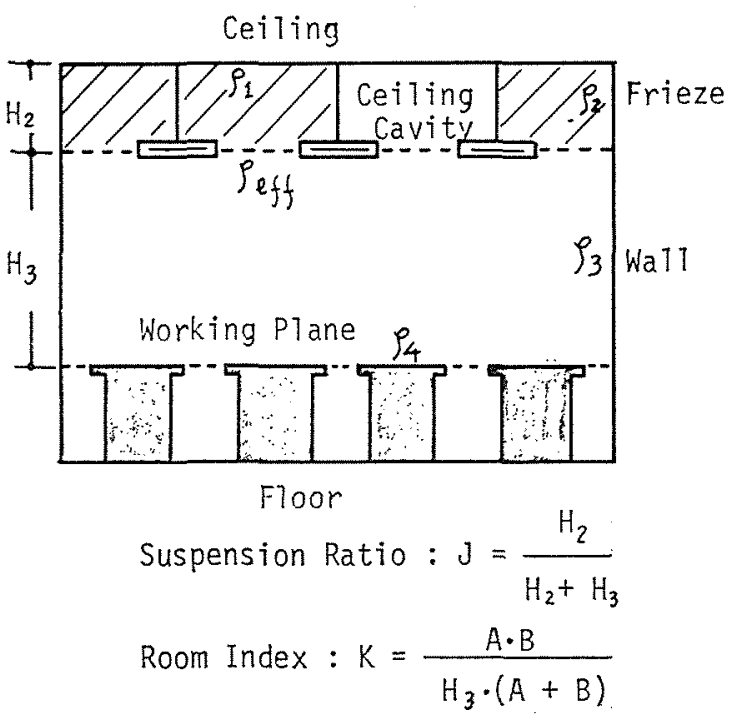

Fig. 1 Cross section of room.

$$
\begin{gathered}
E_{i}=E_{0 i}+\sum_{j=1}^{n} G_{j, i} \cdot \rho_{j} \cdot E_{j} / S_{i} \cdots \ldots \ldots \ldots \ldots \ldots \cdots \cdot(4) \\
\quad(i=1,2,3, \cdots, n)
\end{gathered}
$$

where, $E_{i}, E_{0 i}$ : total and direct mean illuminance

$G_{j, i}=G_{i, j}:$ exchange coefficient between surface: $i$ and $j$

$\rho_{j}:$ reflectance of the surface $: j$

$S_{i}$ : area of the surface: $i$

If the sum of flux on the ceiling and frieze is uniformly distributed over the surface: 1 and 2 , and $E_{1} \doteqdot E_{2}$ holds good, Eq. (4) is transformed by use of utilance as follows ${ }^{4)}$ where $H_{2}=0$. 


$$
\begin{aligned}
&\left(\begin{array}{ccc}
1 & -K / 2 \cdot(1-\alpha) \rho_{3} & -\alpha \rho_{4} \\
1-\rho_{1} & 1-\rho_{3} & 1-\rho_{4} \\
-\alpha \rho_{1} & -K / 2 \cdot(1-\alpha) \rho_{3} & 1
\end{array}\right) \cdot\left(\begin{array}{c}
U_{1} \\
U_{3} \\
U_{4}
\end{array}\right)=\left(\begin{array}{c}
U_{01} \\
1 \\
U_{04}
\end{array}\right) \cdots(5) \\
& \text { where, Room index: } K=\frac{A \cdot B}{H_{3}(A+B)} \\
& \\
& \text { Form factor: } \alpha=F_{1-4} \\
& \text { Total and direct utilance: } U_{i} ; U_{0 i} \\
& U_{i}=S_{i} \cdot E_{i} /(N M) F ; U_{0 i}=S_{i} \cdot E_{0 i} /(N M) F
\end{aligned}
$$

We can easily solve the simultaneous linear equations of Eq. (5) by means of Cramer's formula. For example, after obtaining the total utilance: $U_{4}$, it is possible to replace $U_{4}$ with $S_{4} E_{4} /(N M) F$, and to replace $U_{01} U_{04}$ with $[(N M) F-(N M) F C 4] /$ $(N M) F$ and $F 4 /(N M) F$, respectively. Mean illuminance on the working plane: $E_{4}$ is transformed as the following linear expression which consists of three terms, and three RM coefficients are obtained from Eq. (6).

$$
\begin{aligned}
E_{4}= & \frac{1}{\Delta}\left[\beta\left(1+\alpha \rho_{1}\right)+\alpha\left(1-\rho_{3}\right) \rho_{1}-\beta\left(1-\rho_{1}\right)\right] \frac{(N M) F}{A B} \\
& -\frac{1}{\Delta}\left[\alpha\left(1-\rho_{3}\right) \rho_{1}-\beta\left(1-\rho_{1}\right)\right] \frac{(N M) F C 4}{A B} \\
& +\frac{1}{\Delta}\left[1-\rho_{3}+\beta\left(1-\rho_{1}\right)\right] \frac{F 4}{A B} \cdots \cdots \cdots \cdots \cdots \cdots(6)
\end{aligned}
$$

\begin{tabular}{|c|c|c|}
\hline SURFACE & $R M_{i j}$ & $\begin{array}{l}\text { Coefficients for calculation of mean } \\
\text { illuminance: } E_{i}\end{array}$ \\
\hline \multirow{3}{*}{ CEILING } & $R M_{11}$ & $\frac{1}{d}-\left[\beta\left(1+\alpha \rho_{4}\right)+1-\rho_{3}+\beta\left(1-\rho_{4}\right)\right]$ \\
\hline & $R M_{12}$ & $-\frac{1}{4}-\left[1-\rho_{3}+\beta\left(1-\rho_{4}\right)\right]$ \\
\hline & $R M_{13}$ & $\frac{1}{d}-\left[\alpha\left(1-\rho_{3}\right) \rho_{4}-\beta\left(1-\rho_{4}\right)\right]$ \\
\hline \multirow{3}{*}{ WALIS } & $R M_{31}$ & $\frac{K}{2 d}\left[1-\alpha^{2} \rho_{1} \rho_{4}-\alpha \rho_{1}\left(1-\rho_{4}\right)-\left(1-\rho_{1}\right)\right]$ \\
\hline & $R M_{32}$ & $\frac{K}{2 A}\left[\alpha \rho_{1}\left(1-\rho_{4}\right)+1-\rho_{1}\right]$ \\
\hline & $R M_{33}$ & $-\frac{K}{2 \Delta}\left[\alpha\left(1-\rho_{1}\right) \rho_{4}+1-\rho_{4}\right]$ \\
\hline \multirow{3}{*}{$\begin{array}{l}\text { WORKING } \\
\text { PLAN }\end{array}$} & $R M_{41}$ & $\frac{1}{\Delta}-\left[\beta\left(1+\alpha \rho_{1}\right)+\alpha\left(1-\rho_{3}\right) \rho_{1}-\beta\left(1-\rho_{1}\right)\right]$ \\
\hline & $R M_{42}$ & $-\frac{1}{\Delta}\left[\alpha\left(1-\rho_{3}\right) \rho_{1}-\beta\left(1-\rho_{1}\right)\right]$ \\
\hline & $R M_{43}$ & $\frac{1}{\Delta}-\left[1-\rho_{3}+\beta\left(1-\rho_{1}\right)\right]$ \\
\hline \multicolumn{3}{|c|}{$\begin{aligned} \Delta= & \left(1-\rho_{3}\right)\left(1-\alpha^{2} \rho_{1} \rho_{4}\right)+\beta[\alpha \\
\alpha= & \frac{2}{\pi x y}\left[\log _{e} \sqrt{\frac{\left(1+x^{2}\right)}{1+x^{2}}}\right. \\
& +x \sqrt{1+y^{2}} \tan ^{-1} \frac{x}{\sqrt{1+}} \\
\fallingdotseq & \sqrt{1+K^{-2}}-K^{-1}\end{aligned}$} \\
\hline \multicolumn{3}{|c|}{$\beta=\frac{K}{2}(1-\alpha) \rho_{3}, \quad K=\frac{A \cdot B}{H_{3}(A+B)}$} \\
\hline
\end{tabular}

where, $\beta=K / 2 \cdot(1-\alpha) \rho_{3}$

$\Delta:$ Determinant of coefficients relating to the left part of Eq. (5); $\Delta \neq 0$

The linear expression for calculating mean illuminance on the ceiling and walls are obtained as well

Table 1 Formulas for computing RM coefficients where suspens. on ratio: $J=0$ as Eq. (6). Table 1 shows the computation formulas for $R M$ coefficients where suspension ratio: $J=0$.

\subsection{RM coefficients for suspension ratio : $\mathbf{J} \neq \mathbf{O}$}

In the general lighting installation as shown in Fig. 1, it is noted that the value of mean illuminance contains error by directly applying the computation formulas for $R M$ coefficients in Table 1 . In case of suspension height: $H_{2} \neq 0$, it is possible to calculate the effective reflectance of the luminaire plane: $\rho_{\text {eff }}$ by applying the concept of ceiling cavity. We can approximately and simply calculate the $R M$ coefficients of suspension ratio: $J \neq 0$ by replacing ceiling reflectance: $\rho_{\mathrm{l}}$ with the effective reflectance: $\rho_{\text {eff }}$ in Table 1.

The computation formulas for the effective reflectance have been already proposed by many investigators, and numerical difference of the effective reflectance is quite small as demonstrated in Fig. 2 regardless of various typical computation

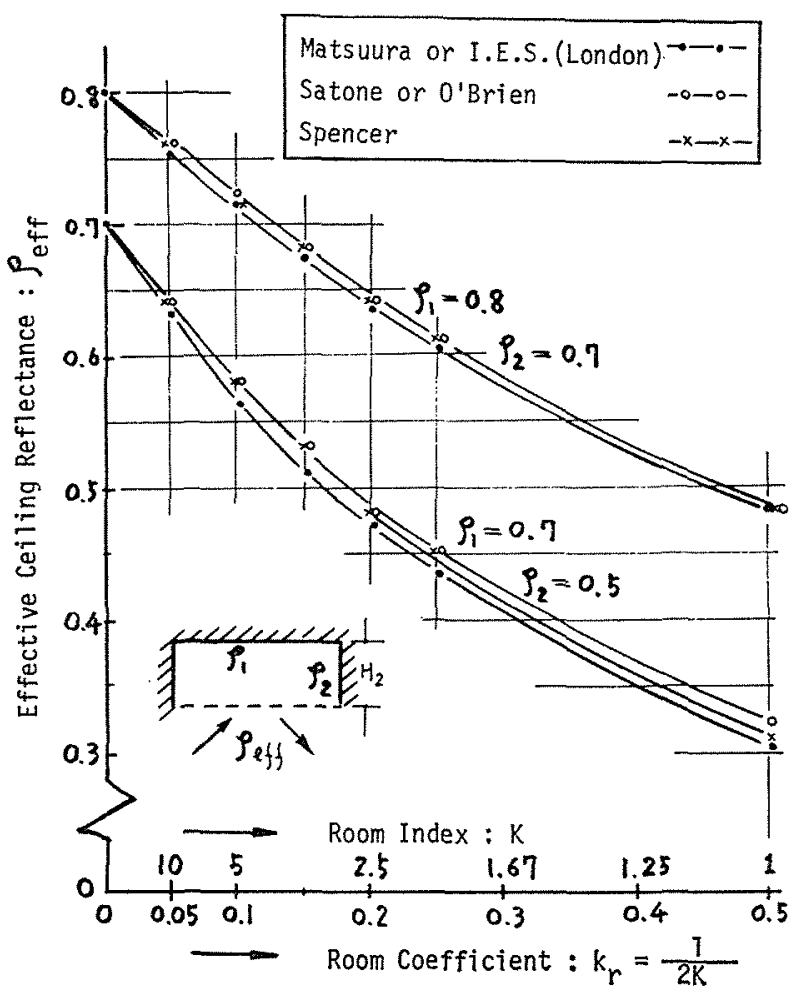

Fig. 2 Effective reflectance for ceiling cavity.

formulas ${ }^{5}$. The detailed computation process and discussion of the effective reflectance are omitted here.

\subsection{MR coefficients}

As $M R$ coefficients are defined from the inverse transformation of the linear expression of $\mathrm{Eq}$. (2), we can directly calculate these coefficients by using the value of $R M$ coefficients or by applying the computation formulas in Table 1 . 


\subsection{RM and MR coefficients based on CIE methods}

The computation formulas based on the CIE methods are different from the method of the authors in terms of computation procedures. The computation methods obtaining the $R M$ and $M R$ coefficients are introduced in Appendix I of "Calculations for Interior Lighting-Basic Method-", and they are not a type of linear expression but a skillful style of matrix expression. The two cases of suspension ratio: $J=0$ and $J \neq 0$ in the above method may be distinguished. Generality of computation procedures of $M R$ and $R M$ coefficients are as follows in case of the suspension ratio: $J \neq 0$.

$$
\boldsymbol{M R}=\left(\begin{array}{llll}
1 & 1 & 1 & 1 \\
0 & 0 & 1 & 0 \\
0 & 0 & 0 & 1
\end{array}\right) \cdot\left(\boldsymbol{C}-\frac{G \cdot R}{A B}\right) \cdot\left(\begin{array}{lll}
1 & 0 & 0 \\
1 & 0 & 0 \\
0 & 1 & 0 \\
0 & 0 & 1
\end{array}\right) \cdots \cdots(7)
$$

$R M=M R^{-1}$

$$
\text { where, }
$$

$$
\begin{aligned}
C & =\left(\begin{array}{cccc}
1 & 0 & 0 & 0 \\
0 & 2 J /(1-J) K & 0 & 0 \\
0 & 0 & 2 / K & 0 \\
0 & 0 & 0 & 1
\end{array}\right), \\
\boldsymbol{G} & =\left(\begin{array}{llll}
G_{11} & G_{12} & G_{13} & G_{14} \\
G_{21} & G_{22} & G_{23} & G_{24} \\
G_{31} & G_{32} & G_{33} & G_{34} \\
G_{41} & G_{42} & G_{43} & G_{44}
\end{array}\right), \quad R=\left(\begin{array}{llll}
\rho_{1} & 0 & 0 & 0 \\
0 & \rho_{2} & 0 & 0 \\
0 & 0 & \rho_{3} & 0 \\
0 & 0 & 0 & \rho_{4}
\end{array}\right)
\end{aligned}
$$

The calculation of coefficients: $G_{i}$, and matrix are quite troublesome unless we can actively use the digital computer in Eq. (7) or Eq. (8). Note that both $\boldsymbol{M R}$ and $\boldsymbol{R} \boldsymbol{M}$ of Eq. (7) and Eq. (8) are $3 \times 3$ matrix, respectively. It is not as simple as it looks in computations.

\section{Roles of $R M$ and $M R$ coefficients}

It is very popular among the existing methods to calculate the mean illuminance on the working plane by using the utilization factor which is supplied by the luminaire manufacturer. $R M$ coefficients are used not only to predict the mean iluminance on the room surface but to calculate the reduced utilance reported by the CIE methods or the utilance on the working plane. Reduced utilance is defined as the ratio of the mean illuminance of surface $i$ to the flux density of installed luminaires, and is obtained from the Eq. (2) by means of the next expression.

$$
\begin{aligned}
& R U_{i}=\frac{E_{i}}{(N M) \cdot F / A B} \\
& =R M_{i 1}+R M_{i 2} \cdot \frac{F C 4}{F}+R M_{i 3} \cdot \frac{F 4}{(N M) \cdot F} \\
& =R M_{i 1}+\left[R M_{i 2}+R M_{i 3} \cdot D R\right] \times . N 4 \cdots \cdots \cdot(9) \\
& (i=1,3,4)
\end{aligned}
$$

Direct ratio: $D R=\frac{F 4}{(N M) \cdot F C 4}$
Lower flux ratio: $. N 4=\frac{F C 4}{F}$

Moreover, utilization factors are obtained from the product of light output ratio of the luminaire and reduced utilance on the working plane: $R U_{4}$.

$U F=$ Light output ratio $\times R U_{4} \cdots \cdots \cdots \cdots \cdots \cdots(10)$ where

Light output ratio: $\eta=$ Flux from luminaire/lamp flux

If one side of $M R$ or $R M$ coefficients is already known, the other side of these coefficients is directly obtained from Eq. (8) by means of inverse matrix.

\section{Computation results of RM and MR coefficients}

In case of the general lighting as shown in Fig. 1, we can easily calculate nine $R M$ coefficients for computation of mean illuminance by directly using the computation formulas of Table 1 for suspension ratio: $J=0$, i.e. suspension height: $H_{2}=0$. As the computation results of these coefficients agree perfectly with the data of CIE methods or AFNOR

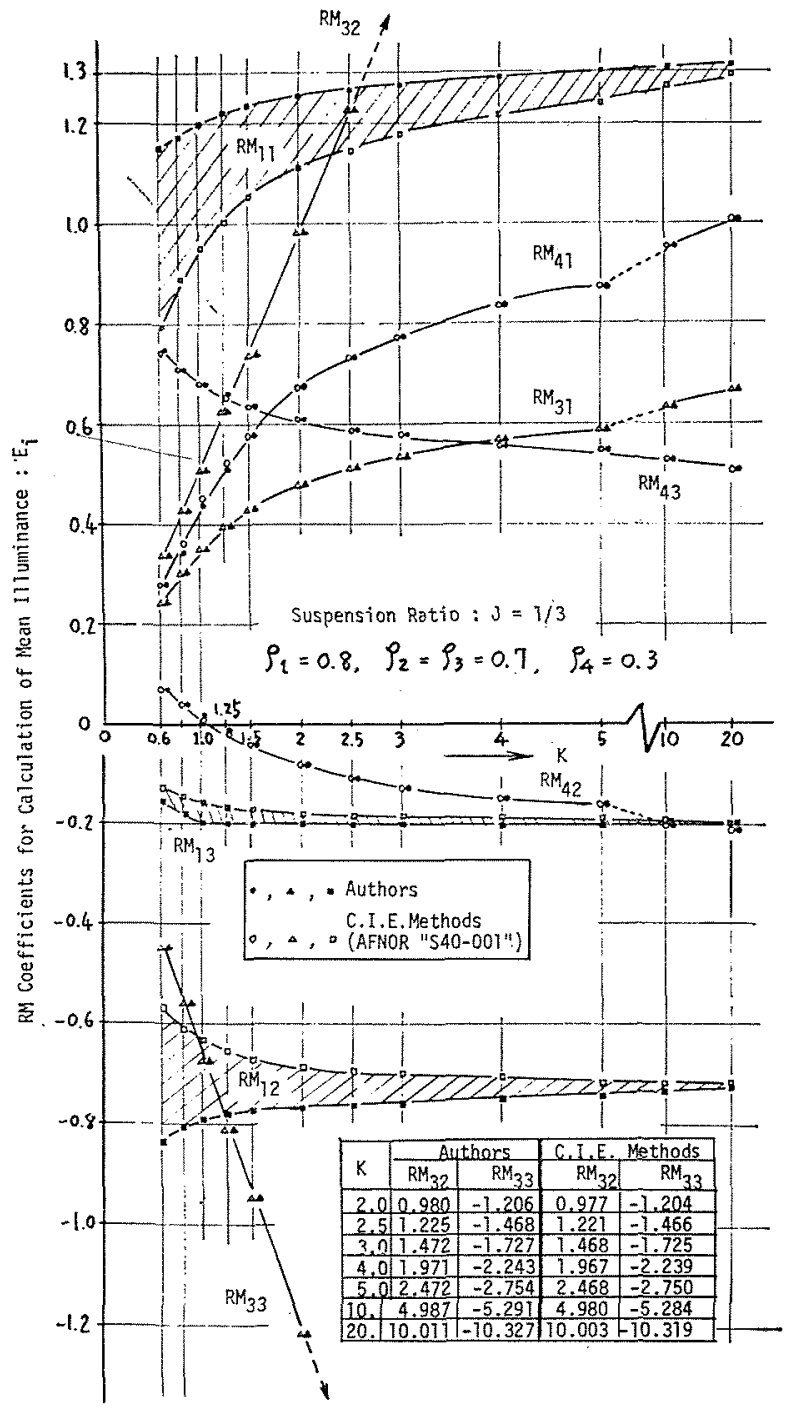

Fig. 3 Nine $R M$ coefficients vs. room index. 
"S40-001"6), we may be sure that the computation procedures and the formulas for $R M$ coefficients which the authors have introduced in above section 3 are correct and useful.

On the other hand, it is necessary to calculate the nine $R M$ coefficients by replacing the ceiling reflecance: $\rho_{1}$ with the effective reflectance of ceiling cavity: $\rho_{\text {eff }}$ in Table 1 for suspension ratio: $J \neq 0$, i.e. suspension height: $H_{2} \neq 0$.

Fig. 3 shows the computation results for nine $R M$ coefficients vs. room index. The data of CIE methods coincide with those of AFNOR "S40-001" except for the significant figure of the last unit. Three $R M$ coefficients for the ceiling obtained by the authors differ from the data of CIE methods or AFNOR "S40-001" for the small room index. This is due to the introduction of concept of ceiling cavity. As $R M_{33}$ and $R M_{34}$ coefficient tend to increase rapidly in proportion to the increase of room index, only part of the curve is demonstrated, and the numerical results are compared with each other in the lower part of Fig. 3.

Fig. 4 (a), (b), (c) are the computation results for nine $R M$ coefficients vs. suspension ratio by keeping the room index and the surface reflectance constant. The change of surface reflectance more or less has influence on $R M_{11}, R M_{31}$ and $R M_{41}$ coefficients, and all the $R M$ coefficients are proportional to suspension ratio. The computation formulas for $R M$ coefficients consist of the sum of two or three terms as described in Table 1. $R M_{11}, R M_{32}$ and $R M_{43}$ coefficients always take the positive value, and $R M_{12}$ and $R M_{33}$ coefficients always take the negative value, because the determinant of Eq. (6) or delta of Table 1 is always positive.

Table 2 shows the results of comparison of the effective reflectance: $\rho_{\text {eff }}$, DELTA : $\Delta$ and $R M$ coefficients by using the various computation formulas for obtaining the $\rho_{\text {eff }}$. Note that difference of $\rho_{e f f}$ is of no consequence for practical purposes.

Fig. 5 demonstrates the relation of three $M R$ coefficients vs. room index for the same parameter

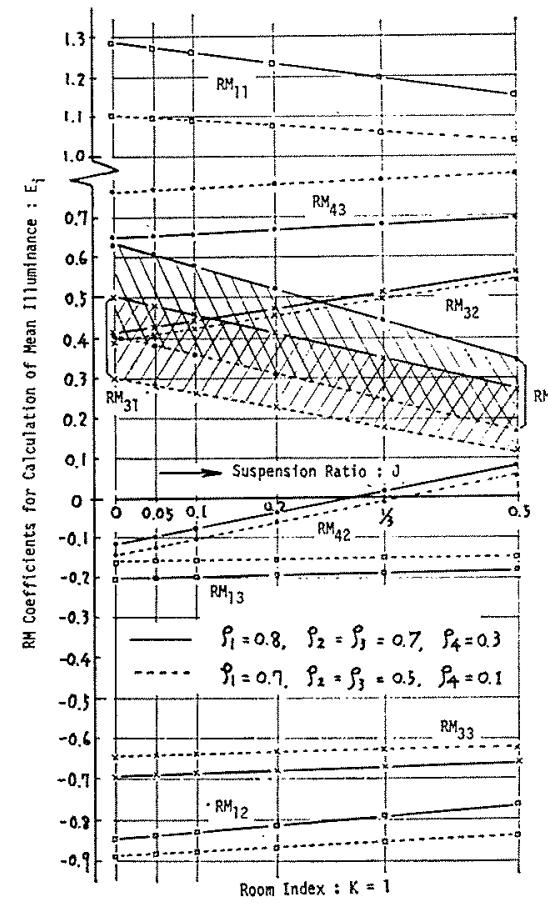

(a)

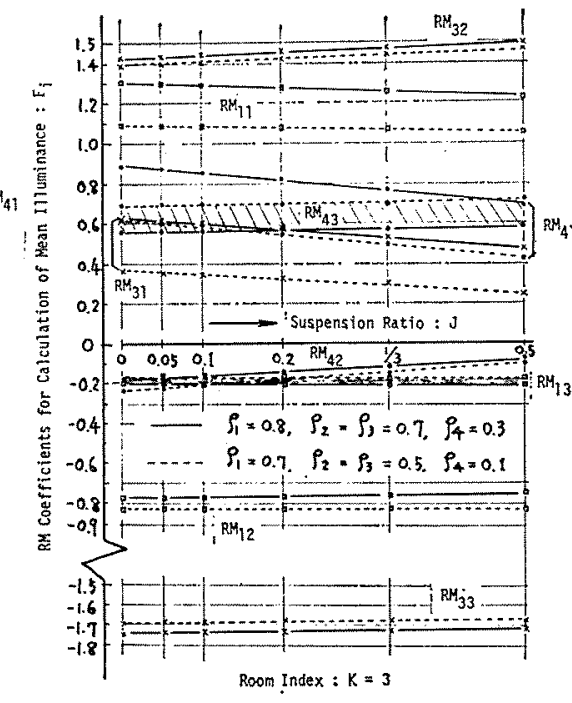

(b)

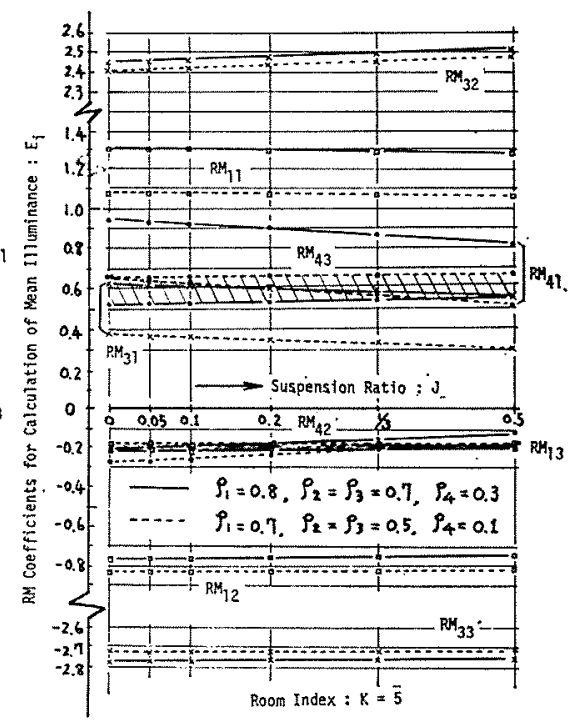

(c)

Fig. 4 Nine $R M$ coefficients vs. suspension ratio.

Table 2 Comparison $\rho_{e f f}, D E L T A$, and RM Coefficients for Suspension Ratio: $J=1 / 3$

\begin{tabular}{|c|c|c|c|c|c|c|c|c|c|c|c|c|c|c|c|c|}
\hline \multicolumn{2}{|c|}{ Room Index } & \multirow{2}{*}{$\rho$} & \multirow{2}{*}{$\rho_{2}$} & \multirow{2}{*}{$\rho_{e f f} *$} & \multirow{2}{*}{$\rho_{3}$} & \multirow{2}{*}{$\rho_{4}$} & \multirow{2}{*}{ DELTA } & \multicolumn{3}{|c|}{ CEILING } & \multicolumn{3}{|c|}{ WALLS } & \multicolumn{3}{|c|}{ WORKING PLANE } \\
\hline$K$ & & & & & & & & $R M_{11}$ & $R M_{12}$ & $R M_{13}$ & $R M_{31}$ & $R M_{32}$ & $R M_{33}$ & $R M_{41}$ & $R M_{42}$ & $R M_{43}$ \\
\hline \multirow{3}{*}{1} & (a) & & & 0.600 & \multirow{3}{*}{0.7} & \multirow{3}{*}{0.3} & 0.562 & 1.199 & -0.789 & -0.189 & 0.351 & 0.511 & -0.677 & 0.442 & 0.013 & 0.680 \\
\hline & (b) & 0.8 & 0.7 & 0.610 & & & 0.560 & 1.203 & -0.792 & -0.189 & 0.358 & 0.507 & -0.661 & 0.451 & 0.007 & 0.678 \\
\hline & (c) & & & 0.607 & & & 0.560 & 1.202 & -0.791 & -0.189 & 0.356 & 0.508 & -0.668 & 0.449 & 0.008 & 0.679 \\
\hline \multirow{3}{*}{3} & (a) & & & 0.721 & \multirow{3}{*}{0.7} & \multirow{3}{*}{0.3} & 0.661 & 1.269 & -0.751 & -0.198 & 0.534 & 1.472 & -1.727 & 0.770 & -0.121 & 0.572 \\
\hline & (b) & 0.8 & 0.7 & 0.728 & & & 0.659 & 1.272 & -0.753 & -0.198 & 0.540 & 1.468 & -1.728 & 0.780 & -0.127 & 0.571 \\
\hline & (c) & & & 0.727 & & & 0.660 & 1.272 & -0.753 & -0.198 & 0.539 & 1.469 & -1.728 & 0.778 & -0.126 & 0.571 \\
\hline \multirow{3}{*}{5} & (a) & & & 0.751 & \multirow{3}{*}{0.7} & \multirow{3}{*}{0.3} & 0.691 & 1.287 & -0.740 & -0.199 & 0.586 & 2.472 & -2.754 & 0.870 & -0.160 & 0.543 \\
\hline & (b) & 0.8 & 0.7 & 0.755 & & & 0.690 & 1.289 & -0.742 & -0.199 & 0.591 & 2.470 & -2.755 & 0.877 & -0.164 & 0.542 \\
\hline & (c) & & & 0.755 & & & 0.690 & 1.289 & -0.742 & -0.199 & 0.591 & 2.470 & -2.755 & 0.877 & -0.164 & 0.542 \\
\hline
\end{tabular}

* Note: See reference (5) for the various computation formulas.

(a) Mastuura or I. E.S. (London), (b) Satone or O'Brien, (c) Spencer 


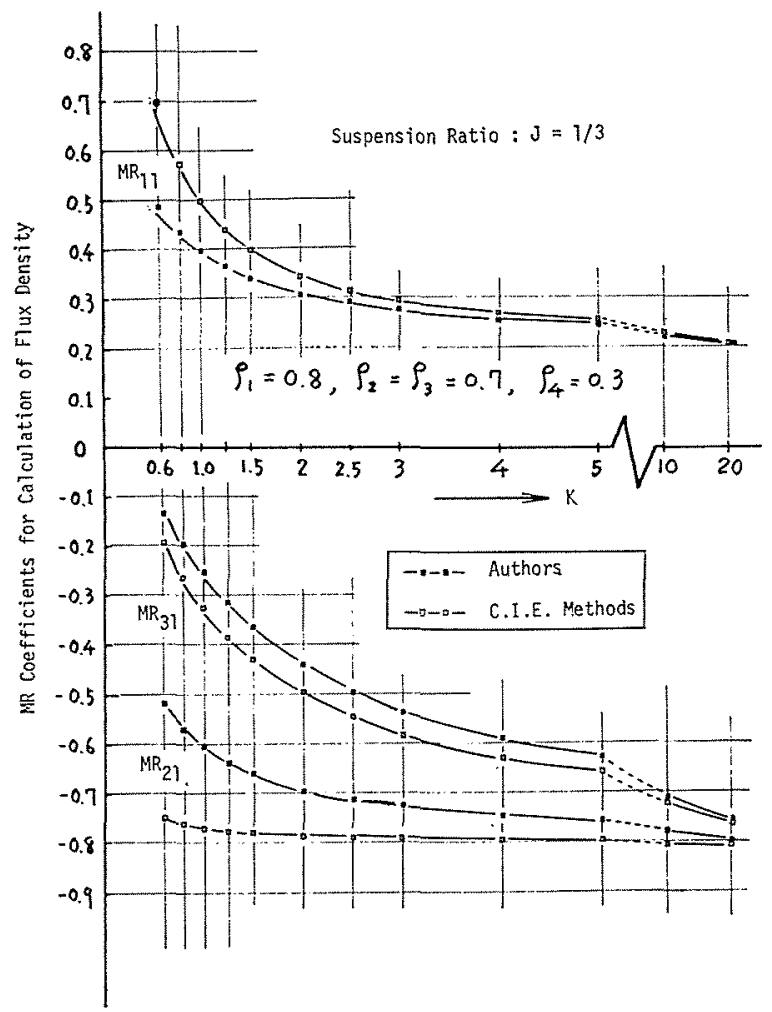

Fig. 5 Three MR coefficients vs. room index.

in Fig. 3. Only $M R_{11}, M R_{21}$ and $M R_{31}$ coefficients differ between the data of authors and that of CIE method because of the introduction of $\rho_{\text {eff }}$ as well as Fig. 3. But the other six coefficients agree with each other. Owing to the inverse transformation, values of the three $R M$ coefficients have an effect on the three $M R$ coefficients as shown in Fig. 5.

Fig. 6 demonstrates the $R M$ coefficients for computing the utilance on the working plane. The two

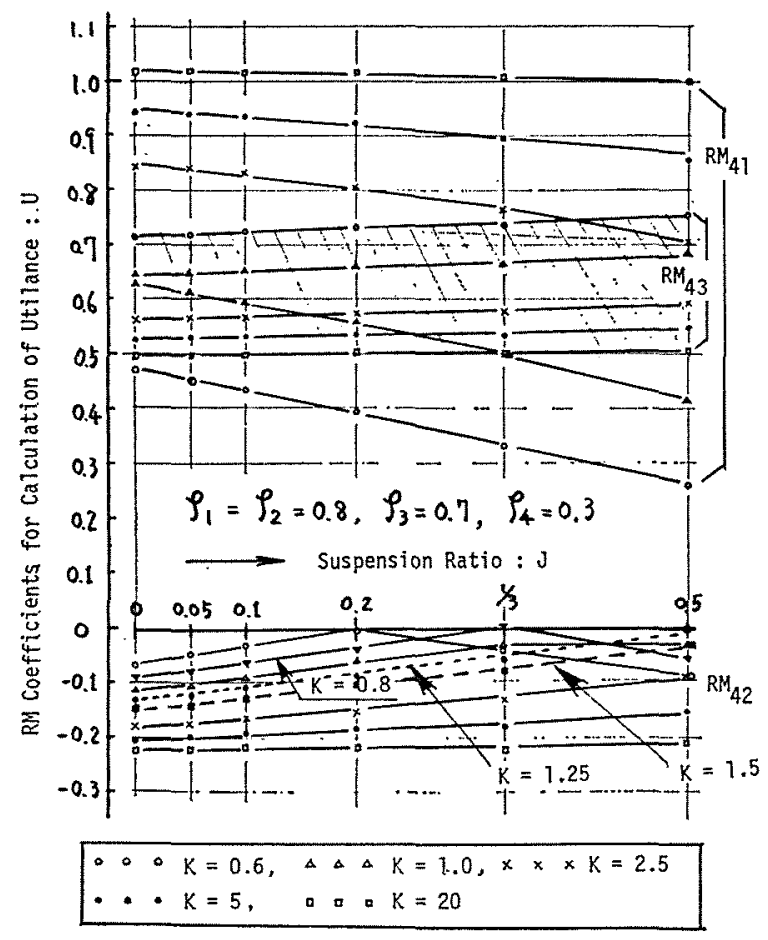

Fig. 6 Three RM coefficients vs. suspension ratio.

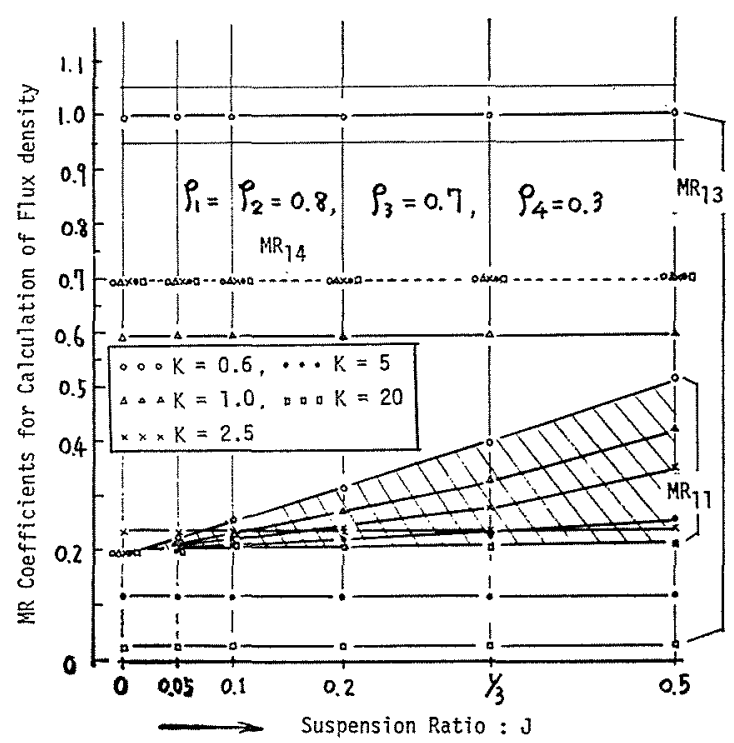

Fig. 7 Three MR coefficients vs. suspension ratio.

$R M$ coefficients are proportional to suspension ratio except for $R M_{42}$ coefficient in which room index is over 1.25 .

Fig. 7 demonstrates the $M R$ coefficients for computing the flux density for installed luminaire, and only $M R_{14}$ coefficient is constant regardless of the reflectance, room index and suspension ratio.

\section{Application to Practical Lighting Design}

Let us consider the practical lighting design in order to explain the use of $R M$ and $M R$ coefficients for CIE methods. Fig. 8 shows the sketch of projected ceiling and across section of the room for general lighting. When the room is lighted by means of the luminaires with two lamps of 2000

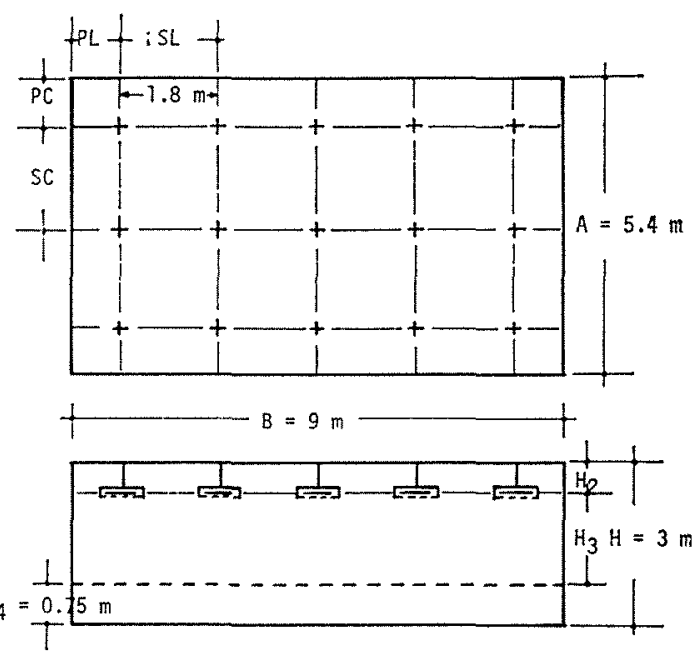

Fig. 8 Sketch of general lighting installation. Proximities: $P L=P C=0.9(\mathrm{~m})$

Spacings: $S L=S C=1.8(\mathrm{~m})$ $\mathrm{H}_{2}=0.56(\mathrm{~m}), \mathrm{H}_{3}=1.69(\mathrm{~m})$

Numbers of total luminaire: $N M=15$

Suspension ratio: $J=0.25$

Room Index: $K=2$ 
( $(\mathrm{m})$, and with CIE flux code such as 56, 83, 95, 100 and 64 , it is possible to calculate the mean illuminance on each room surface by using the procedures of CIE methods as outlined in section 2 . The reflectance of ceiling, frieze, walls and working plane are $0.7,0.5,0.5,0.1$, respectively. The total number of luminaire are 15 (unit), and the working plane is taken to be at $0.75(\mathrm{~m})$ above the floor.

Table 3 shows the results of comparison of mean

Table 3 Comparison of mean illuminance on room surface for general lighting, (See Fig. 8) Numbers of luminaire: $N M=$ 15; Flux of lamp: $2000 \times 2(\mathrm{~lm}) \mathrm{CIE}$ flux code: $56,83,95,100,64$

\begin{tabular}{|c|c|c|c|c|c|c|c|c|}
\hline \multicolumn{3}{|c|}{$\begin{array}{l}\text { Method } \\
\text { Parameter }\end{array}$} & \multicolumn{3}{|c|}{$\begin{array}{l}\text { Result obtained } \\
\text { by authors }\end{array}$} & \multicolumn{3}{|c|}{$\begin{array}{l}\text { Result by using } \\
\text { CIE data }\end{array}$} \\
\hline$K$ & $J^{*}$ & $\rho_{i}$ & $E_{1}(\mathrm{~lx})$ & $E_{3}(\mathrm{~lx})$ & $E_{4}(1 \mathrm{x})$ & $E_{1}(\mathrm{~lx})$ & $E_{3}(1 \mathrm{x})$ & $E_{4}(\mathrm{~lx})$ \\
\hline 1.5 & 0 & $\rho_{1}=0.7$ & 105 & 310 & 613 & 105 & 310 & 613 \\
\hline 2 & 0 & $\rho_{4}=0.1$ & 100 & 318 & 668 & 100 & 318 & 668 \\
\hline 2 & $1 / 3$ & $\begin{array}{l}\rho_{1}=0.7 \\
\rho_{2}=0.5 \\
\rho_{3}=0.5 \\
\rho_{4}=0.1\end{array}$ & 98 & 309 & 655 & 80 & 309 & 655 \\
\hline 2 & $1 / 3$ & $\begin{array}{l}\rho_{1}=0.8 \\
\rho_{2}=0.8 \\
\rho_{3}=0.7 \\
\rho_{4}=0.3\end{array}$ & 279 & 478 & 988 & 252 & 478 & 987 \\
\hline
\end{tabular}

* Note: Numerical data of RM coefficients for ceiling differ between the results of authors and that of CIE in suspension ratio: $J=1 / 3$.

illuminance on each room surface, and the numerical influence of $R M$ coefficients based on data obtained by the authors and data of CIE methods are very few except for mean illuminance on the ceiling. It should be noted that the direct flux on the working plane is calculated by using GM coefficients and $\mathrm{Eq}$. (1), and the mean illuminance on each room surface is calculated by using $R M$ coefficients and Eq. (2).

Table 4 is the comparison of results obtained by the various methods in order to discuss the extent of agreement between the various methods in case of the general lighting of Fig. 8. These data are obtained by using interpolation between the suspension ratio: $J=0$ and $J=1 / 3$. "Average of Basic

Table 4 Comparison of final results where room index: $K=2$ and suspension ratio: $J=$ 0.25

\begin{tabular}{l||c|c|c}
\hline \multicolumn{1}{|c|}{ Various Methods } & $\begin{array}{c}\text { Ceiling } \\
E_{1}(\mathrm{~lx})\end{array}$ & $\begin{array}{c}\text { Walls } \\
E_{3}(\mathrm{~lx})\end{array}$ & $\begin{array}{c}\text { Working Plane } \\
E_{4}(\mathrm{~lx})\end{array}$ \\
\hline Result by authors & 98 & 311 & 658 \\
\hline $\begin{array}{l}\text { Result by CIE method } \\
\begin{array}{l}\text { Average of Basic and } \\
\text { applied method }\end{array}\end{array}$ & 85 & 311 & 658 \\
\hline British method & 99 & 309 & 661 \\
\hline French method & 82 & 310 & 662 \\
\hline German method & 82 & 290 & 658 \\
\hline
\end{tabular}

Note: Results of last four methods are based on Example. 3 of "CIE Applied Method". and Applied method" corresponds to the value of arithmetic mean for three results: (1) Basic Method, (2) Applied Method (not using classification) and (3) Applied Method (using installation classification).

As the concept and computation procedure of British $^{(1)}$, French ${ }^{8)}$ and German Methods ${ }^{9 /}$ are omitted here, the lighting engineers who take an interest in these computation methods should refer to the publications listed in the reference section of this paper.

\section{Conclusion}

The authors have discussed the role and characteristics of $R M$ and $M R$ coefficients by varying the parameters such as room index, suspension ratio and surface reflectance, and have come to the following conclusion by applying the data of these coefficients to practical lighting design.

(1) $R M$ and $M R$ coefficients for the suspension ratio: $J \neq 0$ can be obtained from the basic computation formulas and the concept of effective refiectance.

(2) $R M$ and $M R$ coefficients are generally proportional to the suspension ratio, provided that room index and surface reflectance are fixed.

(3) It is possible to estimate the value of $R M$ or $M R$ coefficients and the final results of mean illuminance or flux density by means of interpolation from the data based on both $J=0$ and $J=1 / 3$ within the practical lighting condition.

\section{Acknowledgements}

The authors would like to acknowledge Dr. R. Kurosawa of Lighting Design Laboratory and Dr. $H$. Satone of Daido Institute of Technology for their valuable advice and discussion.

\section{Reference}

(1) R. Kurosawa: News of Japanese National Committee of CIE, No. 7 (1970) 2; No. 12 (1971) 3; No. 19 (1973) 29; No. 34 (1977) 11 .

M. lizuka and $K$. Ishino: Journal of the Illuminating Engineering Institute of Japan, 60, 5 (1976) 445.

(2) CIE Publication No, 40: Calculation for Interior Lighting -Basic Method-, (1978).

(3) To be published in 1979 CIE Publication No. : Calculation for Interior Lighting -Applied Method-.

(4) M. Iizuka and K. Ishino: Bulletin of Nagoya Institute of Technology, 28 (1976) 323.

(5) M. Iizuka and K. Ishino: Journal of the Illuminating Engineering Institute of Japan, 50, 10 (1966) 550.

(6) Norme Expérimentale S40-001 de l'AFNOR, Paris, (1968): Éclairage artificiel en espace clos; NF $\$ 40-001$, revised edition (1974).

(7) Technical Report No. 2 of the I.E.S. London: The cal culation of Utilization Factors, (1961)

(8) Norme de 1'UTE 71-121, Paris (1970): Méthods simplifiée de prédétermination des éclairements dans les espaces clos et classification correspondente des luminaires; U $\Gamma E$ C71-121, revised edition (1976).

(9) LiTG e. V., (1973), Karlsruhe: Projectierung von Beleuchtungsanlagen für Innerräume; Lichttechnik, 28-8 (1976) Berlin: Die Erweiterung des LiTG Wirkungsgradverfah. rens.

Received 27 Mar. 1979; Revision Received 15 Jun. 1979 